\title{
Diffusive grain-surface chemistry involving the atoms and diatomic molecules of two elements
}

\author{
J. G. L. Rae ${ }^{1}$, N. J. B. Green ${ }^{2}$, T. W. Hartquist ${ }^{1}$, M. J. Pilling ${ }^{3}$, and T. Toniazzo ${ }^{1,4}$ \\ 1 Department of Physics and Astronomy, University of Leeds, Leeds LS2 9JT, UK \\ 2 Chemistry Department, Kings College London, London WC2R 2LS, UK \\ 3 School of Chemistry, University of Leeds, Leeds LS2 9JT, UK \\ ${ }^{4}$ Met. Office, London Road, Bracknell, Berkshire, RG12 2SZ, UK
}

Received 6 November 2002 / Accepted 12 March 2003

\begin{abstract}
A model of the grain surface chemistry involving the accretion of atoms of two different elements, $\mathrm{X}$ and $\mathrm{Y}$, and their reactions to form species $\mathrm{X}_{2}, \mathrm{XY}$, and $\mathrm{Y}_{2}$ was examined for a wide range of choices for the values of its three free parameters - the accretion rate of $\mathrm{X}$ and $\mathrm{Y}$, the desorption rate of $\mathrm{X}$ and the grain surface sweeping time of $\mathrm{Y}$, all considered relative to the grain surface sweeping rate of $X$. Relative production rates of the diatomics were calculated with five methods involving, respectively, a high-order truncation of the master equation, a low-order truncation of the master equation, the standard deterministic rate equation approach, a modified rate equation approach and a set of approximations which are in some cases appropriate for accretion dominated chemistry. The accuracies of the relative production rates calculated with the different methods were assessed for the wide range of model parameters. The more accurate of the low-truncation master equation calculations and the standard deterministic rate equation approach gives results which are in most cases within ten or twenty per cent of the results given by the high-truncation master equation calculations. For many cases, the more accurate of the low order truncation and the standard deterministic rate equation approaches is indicated by a consideration of the average number of atoms of the two species on the grain's surface.
\end{abstract}

Key words. astrochemistry - molecular processes - ISM: clouds - dust, extinction - ISM: molecules

\section{Introduction}

If species accrete from the gas phase onto the surfaces of grains more quickly than they diffuse across a grain surface and react with each other, the surface chemistry is said to be taking place in the reaction limit. Such chemistry has been treated by Pickles \& Williams (1977), who used a system of rate equations similar to those employed in gas-phase chemistry. This approach is often referred to as the standard deterministic rate equation method.

In some cases, species diffuse across grain surfaces and react with each other faster than they accrete. Therefore, when a species is accreted, and there is already another reactive species on the grain, they are likely to react before another particle accretes. The rates of reaction are therefore limited by the rates of accretion; the chemistry is said to be occurring in the $a c$ cretion limit. Under these conditions, the average numbers of reactive species on a grain are small, and a stochastic model is required. The first attempts at stochastic modelling of interstellar grain surface chemistry in the accretion limit were made by Allen \& Robinson (1977) and Tielens \& Hagen (1982). The model of Allen \& Robinson (1977) was constructed

Send offprint requests to: J. G. L. Rae, e-mail: jglr@ast.leeds.ac.uk under the assumption that when a molecule is produced in a grain surface reaction, it immediately enters the gas phase; the model was extended by Tielens \& Hagen (1982) to allow for the accumulation of grain mantles. In their model, Tielens \& Hagen (1982) used Monte Carlo techniques to calculate the steady-state concentrations of mantled species. Later, various other authors constructed Monte Carlo models of grain surface chemistry (e.g. Tielens \& Allamandola 1987; Tielens 1995 (unpublished); Charnley et al. 1997; Tielens \& Charnley 1997; Charnley 2001). Charnley (1998) used Monte Carlo methods to solve the master equation (which gives the probability that there is a certain number of particles of each species) governing gas-phase chemistry, and suggested that a similar approach may be applicable to grain surface chemistry.

As Monte Carlo simulations are computationally too expensive to use in the study of large systems, Caselli et al. (1998, 2002) and Shalabiea et al. (1998) introduced semi-empirical modifications to the standard determininstic rate equations, with the aim of solving the problem of inaccuracy of rate equation methods in the accretion limit.

Biham et al. (2001) and Green et al. (2001) studied, with a master equation approach, the production rate of $\mathrm{H}_{2}$ on surfaces. Green et al. (2001) used a generating function method to obtain an analytic solution for the $\mathrm{H}_{2}$ production rate. 
They also investigated more complex chemistries, using sparse matrix techniques to obtain the probabilities that a grain contains given numbers of particles of each species. With this approach, the calculation must be truncated at a certain maximum number of particles of each species, i.e. there is some truncation value for the number of particles, above which the probabilities are assumed to be zero. With a high enough truncation value, the results are almost exact. Green et al. (2001) used the probabilities obtained in this way to calculate the rates of production of molecules on grain surfaces. They solved the master equation numerically for the system of $\mathrm{H}$ and $\mathrm{O}$, reacting on grain surfaces to form $\mathrm{H}_{2}, \mathrm{O}_{2}$, and $\mathrm{OH}$, and also for the system of $\mathrm{H}, \mathrm{O}$, and $\mathrm{N}$, reacting to form $\mathrm{H}_{2}, \mathrm{O}_{2}, \mathrm{OH}, \mathrm{NH}, \mathrm{N}_{2}, \mathrm{NO}$, $\mathrm{NO}_{2}, \mathrm{H}_{2} \mathrm{O}$, and $\mathrm{NH}_{3}$. They compared the results given by the solution of the master equation with a high truncation value, to those given by (i) the use of the master equation method with a low truncation value; (ii) the standard deterministic rate equation; and (iii) an approximate method, based on the assumption that the sweeping rate of atomic hydrogen on grain surfaces is so great that a reactive species already on the surface of a grain will react as soon as an $\mathrm{H}$ atom is accreted.

Stantcheva et al. (2002) solved the master equation for the system of $\mathrm{H}, \mathrm{O}$, and $\mathrm{CO}$ reacting on the surfaces of grains to produce $\mathrm{O}_{2}, \mathrm{H}_{2}, \mathrm{H}_{2} \mathrm{O}, \mathrm{CO}_{2}, \mathrm{H}_{2} \mathrm{CO}$, and $\mathrm{CH}_{3} \mathrm{OH}$. They investigated the accuracy of results given by the method for different truncation values and also the accuracies of results given by other approaches.

In this paper, we examine the system of atoms $\mathrm{X}$ and $\mathrm{Y}$ reacting to form $\mathrm{X}_{2}, \mathrm{XY}$, and $\mathrm{Y}_{2}$ on grain surfaces. We study a wide variety of adsorption, desorption, and diffusion rates, covering the accretion limit, the reaction limit, and cases intermediate between them. We compare the almost exact results, obtained through the solution of the master equation for high truncation values, with the results obtained (i) through the solution of the master equation with low truncation values; (ii) with the approximate method used by Green et al. (2001); (iii) with the standard deterministic rate equation approach; and (iv) with a modified rate equation approach similar to that employed by Caselli et al. (2002). Although the network we study is smaller than that of Stantcheva et al. (2002), we examine a much larger range of adsorption, desorption, and diffusion rates, thereby gaining insight into the parameter ranges in which each method may be applied.

In Sect. 2, the various approaches to the problem are discussed. We present our results in Sect. 3, and Sect. 4 concludes the paper.

\section{The various approaches to the problem}

We consider a system in which atoms $\mathrm{X}$ and $\mathrm{Y}$ accrete from the gas phase onto the surfaces of grains, diffuse across the grain surfaces, and react with each other to form $\mathrm{X}_{2}, \mathrm{Y}_{2}$, and $\mathrm{XY}$, i.e. in which the following reactions take place:

$\mathrm{X}+\mathrm{X} \rightarrow \mathrm{X}_{2}$
$\mathrm{Y}+\mathrm{X} \rightarrow \mathrm{XY}$
$\mathrm{Y}+\mathrm{Y} \rightarrow \mathrm{Y}_{2}$.
We assume that the $X_{2}, X Y$, and $Y_{2}$ molecules remain on the grain surfaces, but that they do not react with each other or with $\mathrm{X}$ or $\mathrm{Y}$. We allow for desorption of $\mathrm{X}$ from grains, but assume that desorption of $\mathrm{Y}$ is negligible. Let $a_{\mathrm{X}}$ and $a_{\mathrm{Y}}$ be the rates of accretion of $\mathrm{X}$ and $\mathrm{Y}$ respectively, and $d_{\mathrm{X}}$ be the rate of desorption of $\mathrm{X}$. Let $t_{\mathrm{S}}(\mathrm{X})$ and $t_{\mathrm{s}}(\mathrm{Y})$ be the grain surface sweeping times for $\mathrm{X}$ and $\mathrm{Y}$ respectively. To reduce the number of free parameters, we assume

$a_{\mathrm{X}}=a_{\mathrm{Y}}$.

This assumption is likely to be reasonable at some densities in dense cores if $\mathrm{X}$ is hydrogen and $\mathrm{Y}$ is oxygen. Furthermore, in a number of previous studies (e.g. Stantcheva et al. 2002), the ratio of accretion rates has been varied.

We normalise with respect to $t_{\mathrm{s}}(\mathrm{X})$ as follows.

$\alpha=a_{\mathrm{X}} t_{\mathrm{s}}(\mathrm{X})$

$\delta=d_{\mathrm{X}} t_{\mathrm{s}}(\mathrm{X})$

$\tau=\frac{t_{\mathrm{s}}(\mathrm{Y})}{t_{\mathrm{s}}(\mathrm{X})}$

The dimensionless rates for reactions (1) to (3) are

$\kappa_{\mathrm{XX}}=2$

$\kappa_{\mathrm{XY}}=1+\tau^{-1}$

$\kappa_{\mathrm{YY}}=2 \tau^{-1}$.

Equations (8) to (10) may be compared to the rates given by Eq. (9) of Caselli et al. (1998) for two arbitrary species I and J.

The production rates of $\mathrm{X}_{2}, \mathrm{XY}$, and $\mathrm{Y}_{2}$ were calculated with the various approaches for $13 \times 13 \times 13$ sets of values of $\alpha, \delta, \tau$ between 0.1 and 10. Calculations were also performed for $9 \times 13 \times 9$ sets of $0.01 \leq \alpha \leq 0.09,0.1 \leq \delta \leq 10.0$, $0.01 \leq \tau \leq 0.09$.

We now discuss in detail the master equation approach, the standard and modified rate equation approaches, and the approximate method of Green et al. (2001).

\subsection{Master equation approach}

Green et al. (2001) gave the master equation for the hydrogen and oxygen system in their Eq. (29), and the rates of production of $\mathrm{H}_{2}, \mathrm{OH}$, and $\mathrm{O}_{2}$ in their Eqs. (26) to (28). The generalisation of their equations to species $\mathrm{X}, \mathrm{Y}, \mathrm{X}_{2}, \mathrm{XY}$, and $\mathrm{Y}_{2}$ is trivial. The average numbers of $\mathrm{X}$ and $\mathrm{Y}$ atoms on the surface of a grain are:

$$
\begin{aligned}
& \langle N(\mathrm{X})\rangle=\sum_{\substack{i=0 \\
j=0}}^{\infty} i P(i, j) \\
& \langle N(\mathrm{Y})\rangle=\sum_{\substack{i=0 \\
j=0}}^{\infty} j P(i, j)
\end{aligned}
$$

where $P(i, j)$ is the probability that on the surface of a grain there are $i$ atoms of species $\mathrm{X}$ and $j$ atoms of species $\mathrm{Y}$. We assumed the system to be in steady state, so that $\frac{\mathrm{d} P(i, j)}{\mathrm{d} t}=0$ for all $i, j$, and followed Green et al. (2001) in solving the master 
equation through the inversion of a sparse matrix by the rowindexed method. As discussed in Sect. 1, with this approach it is necessesary to truncate the sums at particular values of $i$ and $j$. They were truncated first at $i=j=2$ (which will be referred to as the low truncation case), and then at higher values (which will be referred to as the high truncation case), above which the results did not change appreciably if $i$ and $j$ were increased further. The results given by the master equation approach with high truncation values of $i$ and $j$ - typically $i=j \geq 5$ - can be considered to be exact, and will be referred to as the exact results.

\subsection{Standard rate equation approach}

The standard deterministic rate equations for the $\mathrm{H}, \mathrm{O}, \mathrm{H}_{2}, \mathrm{OH}$, and $\mathrm{O}_{2}$ system are given by Caselli et al. (1998) in their Eqs. (4) to (8). They can be generalised easily to the $\mathrm{X}, \mathrm{Y}, \mathrm{X}_{2}, \mathrm{XY}$, and $\mathrm{Y}_{2}$ system. The rate equations for $\mathrm{X}$ and $\mathrm{Y}$ were integrated with a Gear algorithm until the system reached steady state. Then, the production rates of $\mathrm{X}_{2}, \mathrm{XY}$, and $\mathrm{Y}_{2}$ were calculated from their rate equations.

\subsection{Modified rate equation approach}

We introduced modifications to the rate equations, similar to those suggested by Caselli et al. $(1998,2002)$ in their attempt to develop a set of deterministic equations appropriate in both the reaction limit and the accretion limit. The modifications we used were based on those used by Caselli et al. (2002).

We define $\theta_{1}$ and $\theta_{2}$ as

$\theta_{1}=\frac{\kappa_{\mathrm{X}, \mathrm{X}}\langle N(\mathrm{X})\rangle+\kappa_{\mathrm{X}, \mathrm{Y}}\langle N(\mathrm{Y})\rangle}{\alpha}$

$\theta_{2}=\frac{\kappa_{\mathrm{Y}, \mathrm{Y}}\langle N(\mathrm{Y})\rangle+\kappa_{\mathrm{X}, \mathrm{Y}}\langle N(\mathrm{X})\rangle}{\alpha}$.

In cases in which $\theta_{1} \leq 1$ and $\theta_{2} \leq 1$, the standard deterministic rate equations were used. Otherwise, the equations were modified as follows.

In the case that $\theta_{1}>1$ and $\theta_{2} \leq 1$, the probability that species $\mathrm{Y}$ reacts is either less than or equal to 1 , so that for $\mathrm{Y}_{2}$ formation the standard deterministic rate equation approach can be used. However the probability that species $\mathrm{X}$ reacts is greater than 1 , and the equations for the production of $X_{2}$ and XY must be modified. The rate coefficients $\kappa_{\mathrm{X}, \mathrm{X}}$ and $\kappa_{\mathrm{X}, \mathrm{Y}}$ are replaced by the larger of $\alpha$ and $\delta$ (Caselli et al. 1998, 2002); this quantity will be denoted by $\kappa_{\mathrm{X}}^{\prime}$. In addition, the formation rate of $X_{2}$ is multiplied by the probability that $X$ reacts with another $\mathrm{X}$ instead of with a $\mathrm{Y}$, and the rate of formation of $\mathrm{XY}$ is multiplied by the probability that $\mathrm{X}$ reacts with $\mathrm{Y}$ and not with another $X$ (following Caselli et al. 2002). The production rates, $\Gamma\left(\mathrm{X}_{2}\right)$ and $\Gamma(\mathrm{XY})$, of $\mathrm{X}_{2}$ and $\mathrm{XY}$, in this approach become

$$
\begin{aligned}
\Gamma\left(\mathrm{X}_{2}\right) & =\kappa_{\mathrm{X}}^{\prime}\langle N(\mathrm{X})\rangle \frac{\kappa_{\mathrm{X}, \mathrm{X}}\langle N(\mathrm{X})\rangle}{\kappa_{\mathrm{X}, \mathrm{X}}\langle N(\mathrm{X})\rangle+\kappa_{\mathrm{X}, \mathrm{Y}}\langle N(\mathrm{Y})\rangle} \\
\Gamma(\mathrm{XY}) & =\kappa_{\mathrm{X}}^{\prime}\langle N(\mathrm{X})\rangle \frac{\kappa_{\mathrm{X}, \mathrm{Y}}\langle N(\mathrm{Y})\rangle}{\kappa_{\mathrm{X}, \mathrm{Y}}\langle N(\mathrm{Y})\rangle+\kappa_{\mathrm{X}, \mathrm{X}}\langle N(\mathrm{X})\rangle} .
\end{aligned}
$$

After (15), (16), and the standard rate equation for $\mathrm{Y}_{2}$ are used, $\theta_{2}$ is recalculated; if it is found to exceed unity then the approach described below for cases in which $\theta_{1}>1$ and $\theta_{2}>1$ is applied.

The case in which $\theta_{1} \leq 1$ and $\theta_{2}>1$ is the same as the previous case, except that $\mathrm{X}$ is replaced by $\mathrm{Y}$ and vice versa. So $\Gamma\left(\mathrm{X}_{2}\right)$ can be calculated with the standard determistic rate equation approach, and the rates of formation, $\Gamma(X Y)$ and $\Gamma\left(\mathrm{Y}_{2}\right)$, of $\mathrm{XY}$ and $\mathrm{Y}_{2}$, are

$$
\begin{aligned}
\Gamma(\mathrm{XY}) & =\kappa_{\mathrm{Y}}^{\prime}\langle N(\mathrm{Y})\rangle \frac{\kappa_{\mathrm{X}, \mathrm{Y}}\langle N(\mathrm{X})\rangle}{\kappa_{\mathrm{X}, \mathrm{Y}}\langle N(\mathrm{X})\rangle+\kappa_{\mathrm{Y}, \mathrm{Y}}\langle N(\mathrm{Y})\rangle} \\
\Gamma\left(\mathrm{Y}_{2}\right) & =\kappa_{\mathrm{Y}}^{\prime}\langle N(\mathrm{Y})\rangle \frac{\kappa_{\mathrm{Y}, \mathrm{Y}}\langle N(\mathrm{Y})\rangle}{\kappa_{\mathrm{Y}, \mathrm{Y}}\langle N(\mathrm{Y})\rangle+\kappa_{\mathrm{X}, \mathrm{Y}}\langle N(\mathrm{X})\rangle}
\end{aligned}
$$

where $\kappa_{\mathrm{Y}}^{\prime}$ is the larger of the accretion rate and the desorption rate of $\mathrm{Y}$; however because we are neglecting desorption of $\mathrm{Y}$, $\kappa_{\mathrm{Y}}^{\prime}=\alpha$. After Eqs. (17) and (18) and the standard rate equation for $\mathrm{X}_{2}$ production are used, $\theta_{1}$ is recalculated; if it is found to be greater than unity then the approach described below for cases in which $\theta_{1}>1$ and $\theta_{2}>1$ is applied.

In the case in which both $\theta_{1}$ and $\theta_{2}$ are greater than 1 , the rate equations for $\mathrm{X}_{2}, \mathrm{XY}$, and $\mathrm{Y}_{2}$ must be modified. For the formation rates of $X_{2}$ and $Y_{2}$ we used Eqs. (15) and (18), respectively. For the XY formation rate, we used Eq. (16) when $\theta_{1}>\theta_{2}$, and Eq. (17) when $\theta_{1}<\theta_{2}$.

In all cases, the rates of change of $\langle N(\mathrm{X})\rangle$ and $\langle N(\mathrm{Y})\rangle$ are

$$
\begin{aligned}
& \frac{\mathrm{d}\langle N(\mathrm{X})\rangle}{\mathrm{d} t}=\alpha-\delta\langle N(\mathrm{X})\rangle-2 \Gamma\left(\mathrm{X}_{2}\right)-\Gamma(\mathrm{XY}) \\
& \frac{\mathrm{d}\langle N(\mathrm{Y})\rangle}{\mathrm{d} t}=\alpha-2 \Gamma\left(\mathrm{Y}_{2}\right)-\Gamma(\mathrm{XY}) .
\end{aligned}
$$

Equations (19) and (20) were integrated with a Gear algorithm until the system reached steady state. At each step in the integration, the rates of production of $\mathrm{X}_{2}, \mathrm{XY}$, and $\mathrm{Y}_{2}$ were calculated using the standard deterministic, or modified, rate equations as appropriate, and were used in the calculations of $\frac{\mathrm{d}\langle N(\mathrm{X})\rangle}{\mathrm{d} t}$ and $\frac{\mathrm{d}\langle N(\mathrm{Y})\rangle}{\mathrm{d} t}$.

\subsection{Approximate method}

Green et al. (2001) introduced an analytic approximation to the master equation method, based on the assumption that the sweeping rate of $\mathrm{X}$ is so fast that if there is a reactive species ( $\mathrm{X}$ or $\mathrm{Y}$ in this case) on the surface of a grain, a $\mathrm{X}$ atom accreting onto the grain will react immediately. This is equivalent to assuming that $\kappa_{\mathrm{X}, \mathrm{X}} \gg \alpha$, and that the probability of there being more than one reactive species on the surface of a grain is small. Green et al. (2001) showed that, under these assumptions

$$
\begin{aligned}
& \Gamma\left(\mathrm{X}_{2}\right) \approx \frac{\alpha^{2} P(0,0)}{2 \alpha+\delta} \\
& \Gamma(\mathrm{XY}) \approx \alpha^{2}\left[\frac{1}{2 \alpha+\delta}+\frac{1}{2 \alpha}\right] P(0,0) \\
& \Gamma\left(\mathrm{Y}_{2}\right) \approx \frac{\kappa_{\mathrm{Y}, \mathrm{Y}}}{\kappa_{\mathrm{Y}, \mathrm{Y}}+2 \alpha} \frac{\alpha}{2} P(0,0)
\end{aligned}
$$

where

$P(0,0) \approx\left[\frac{3}{2}+\frac{\alpha}{2 \alpha+\delta}\right]^{-1}$. 


\section{Results and discussion}

For each of the methods described in Sect. 2, the production rates of $\mathrm{X}_{2}, \mathrm{XY}$, and $\mathrm{Y}_{2}$ were evaluated with $\alpha, \delta, \kappa_{\mathrm{X}, \mathrm{X}}, \kappa_{\mathrm{X}, \mathrm{Y}}$, and $\kappa_{\mathrm{Y}, \mathrm{Y}}$ given by Eqs. (5) to (10), with $\alpha, \delta$, and $\tau$ having various values, as described in Sect. 2.

Following Caselli et al. (1998), we calculated the relative abundances of $\mathrm{X}_{2}, \mathrm{XY}$, and $\mathrm{Y}_{2}$ on the surfaces of grains as

$$
\begin{aligned}
& x\left(\mathrm{H}_{2}\right)=\frac{\Gamma\left(\mathrm{X}_{2}\right)}{\Gamma\left(\mathrm{X}_{2}\right)+\Gamma(\mathrm{XY})+\Gamma\left(\mathrm{Y}_{2}\right)} \\
& x(\mathrm{XY})=\frac{\Gamma(\mathrm{XY})}{\Gamma\left(\mathrm{X}_{2}\right)+\Gamma(\mathrm{XY})+\Gamma\left(\mathrm{Y}_{2}\right)} \\
& x\left(\mathrm{O}_{2}\right)=\frac{\Gamma\left(\mathrm{Y}_{2}\right)}{\Gamma\left(\mathrm{X}_{2}\right)+\Gamma(\mathrm{XY})+\Gamma\left(\mathrm{Y}_{2}\right)} .
\end{aligned}
$$

We calculated the difference between the exact results, and the results obtained (i) through the solution of the master equation for the low-truncation case; (ii) with the standard rate equation approach; (iii) with the modified rate equation approach; and (iv) with the approximate method. The results are plotted against $\alpha$ and $\delta$, for $\tau=1.0$, in Figs. 1 to 4 .

Figures 1 to 4 show the magnitudes of the percentage discrepancies between: the results given by the standard rate equation approach, and the exact results (Fig. 1); the results given by the modified rate equation approach, and the exact results (Fig. 2); the results given by the approximate method, and the exact results (Fig. 3); and the results given by the master equation approach for the low truncation case, and the exact results (Fig. 4).

Due to lack of space, the results are plotted against $\alpha$ and $\delta$ for $\tau=1.0$ only. Results for other values of $\tau$ can be found at http://ast. leeds.ac.uk/ jglr. In each of Figs. 1 to 4, plot (a) shows the discrepancies in $x\left(\mathrm{X}_{2}\right)$; plot (b) shows the discrepancies in $x(\mathrm{XY})$; and plot (c) shows the discrepancies in $x\left(\mathrm{Y}_{2}\right)$.

In Fig. 5, the percentage discrepancies are plotted against $\langle N(\mathrm{X})\rangle$ and $\langle N(\mathrm{Y})\rangle$, the average numbers of $\mathrm{X}$ and $\mathrm{Y}$ atoms on the surface of a grain, as given by the exact method and Eqs. (11) and (12). The white gaps at the top left-hand and bottom left-hand corners of the plots in Fig. 5 are caused by the fact that we did not explore regions of $\alpha-\delta-\tau$ space that yielded these values of $\langle N(\mathrm{X})\rangle$ and $\langle N(\mathrm{Y})\rangle$. The uneven contours in Fig. 5 are caused by the fact that any point in $\langle N(\mathrm{X})\rangle-\langle N(\mathrm{Y})\rangle$ space does not correspond to a single point in $\alpha-\delta-\tau$ space, and therefore the discrepancies do not depend only on $\langle N(\mathrm{X})\rangle$ and $\langle N(\mathrm{Y})\rangle$. In Fig. 6 the discrepancies are plotted against $\langle N(\mathrm{X})\rangle$ and $\langle N(\mathrm{Y})\rangle$ for $\tau=1.0$ only. Again the white areas are regions of $\langle N(\mathrm{X})\rangle-\langle N(\mathrm{Y})\rangle$ space to which no point in $\alpha-\delta$ space corresponds for $\tau=1.0$. In Figs. 5 and 6 , plots $\mathrm{a}, \mathrm{b}$ and $\mathrm{c}$ are for the standard rate equation approach, plots $\mathrm{d}$, e, and $\mathrm{f}$ for the modified rate equation approach, plots $\mathrm{g}$, $\mathrm{h}$, and $\mathrm{i}$ for the approximate method, and plots $\mathrm{j}, \mathrm{k}$, and $\mathrm{l}$ for the low-truncation case of the master equation approach. Plots a, $\mathrm{d}, \mathrm{g}$, and $\mathrm{j}$ give the percentage discrepancy in $x\left(\mathrm{X}_{2}\right)$, plots $\mathrm{b}, \mathrm{e}$, $\mathrm{h}$, and $\mathrm{k}$ the percentage discrepancy in $x(\mathrm{XY})$, and plots $\mathrm{c}, \mathrm{f}$, $\mathrm{i}$, and 1 the percentage discrepancy in $x\left(\mathrm{Y}_{2}\right)$. In Figs. 5 and 6 , the ranges of $\langle N(\mathrm{X})\rangle-\langle N(\mathrm{Y})\rangle$ are different because there is no desorption of $\mathrm{Y}$, so $\mathrm{X}$ and $\mathrm{Y}$ are not symmetric. a)

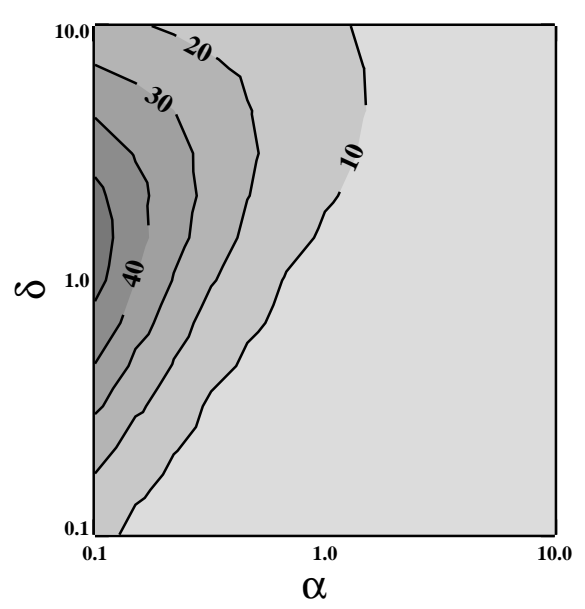

b)

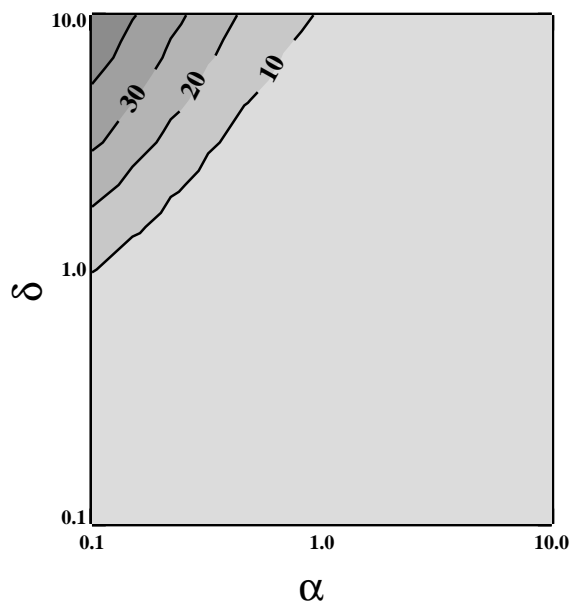

c)

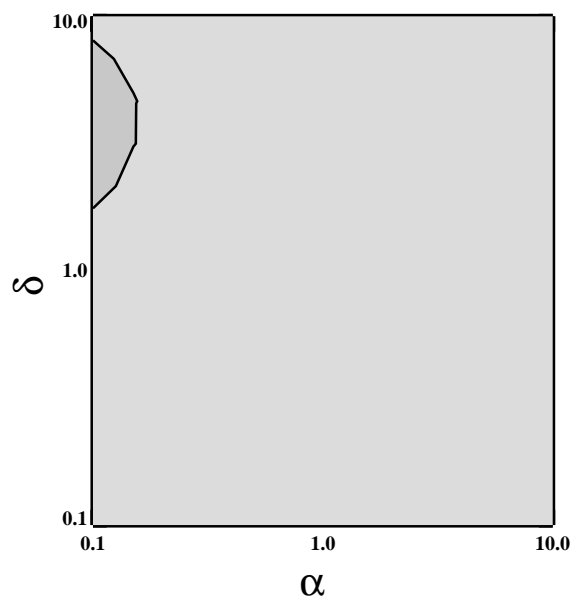

Fig. 1. Percentage discrepancies in results given by standard rate equation approach for $\tau=1.0$. a) Discrepancy in $x\left(\mathrm{X}_{2}\right)$; b) discrepancy in $x(\mathrm{XY})$; c) discrepancy in $x\left(\mathrm{Y}_{2}\right)$. Darker regions indicate greater discrepancies.

In all of Figs. 1 to 6 , lighter regions indicate lower percentage discrepancies, and darker regions higher percentage discrepancies.

Although only the results for $\tau=1.0$ are shown in Fig. 1, it was found that the results given by the rate equation approach are inaccurate for the case of low $\alpha$, high $\delta$, and low $\tau$, 
a)

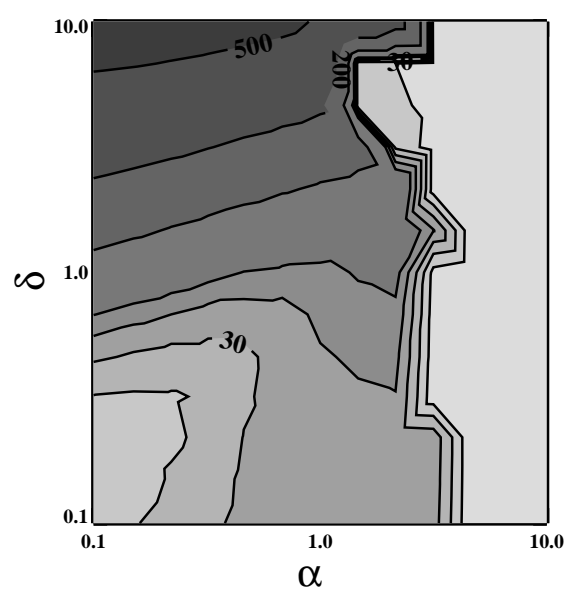

b)

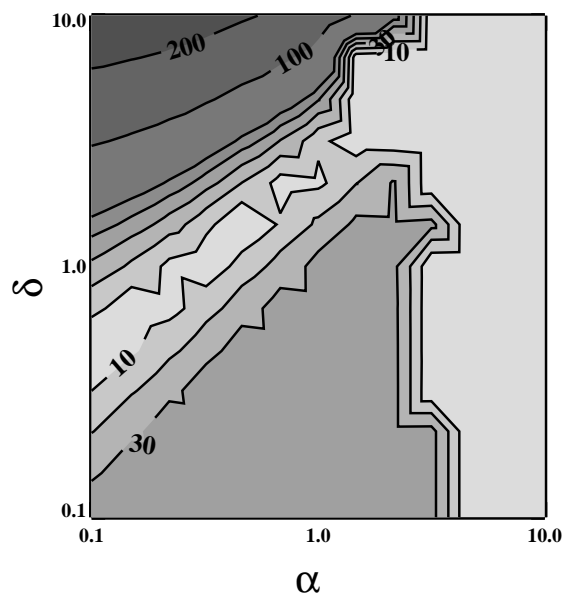

c)

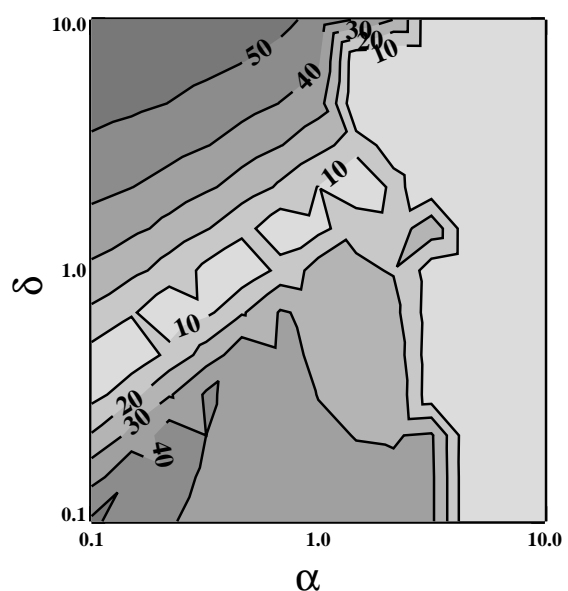

Fig. 2. Percentage discrepancies in results given by modified rate equation approach for $\tau=1.0$. a) Discrepancy in $x\left(\mathrm{X}_{2}\right)$; b) discrepancy in $x(\mathrm{XY})$; c) discrepancy in $x\left(\mathrm{Y}_{2}\right)$. Darker regions indicate greater discrepancies.

corresponding to situations in which the rates of accretion are low and the rates of $\mathrm{X}$ desorption, and of reactions (2) and (3), are high, so that the average surface population on a grain is low. This conclusion is confirmed by Figs. 5a-c and $6 \mathrm{a}-\mathrm{c}$, which show that this approach is least accurate for small $\langle N(\mathrm{X})\rangle$ and $\langle N(\mathrm{Y})\rangle$. These are the conditions under which it is known that the rate equation approach breaks down. a)

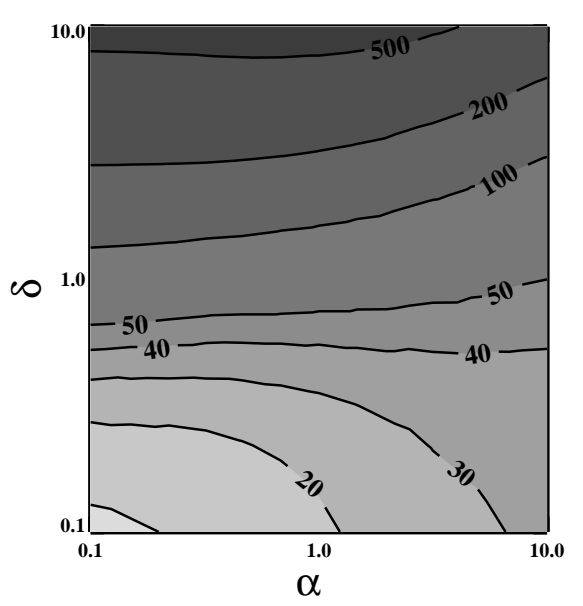

b)

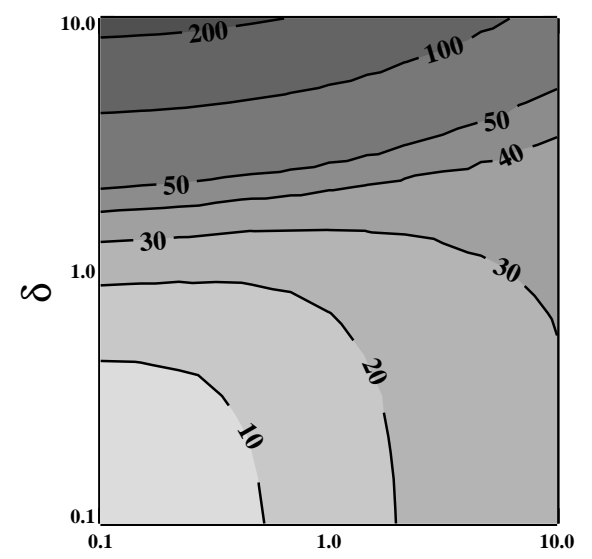

$\alpha$

c)

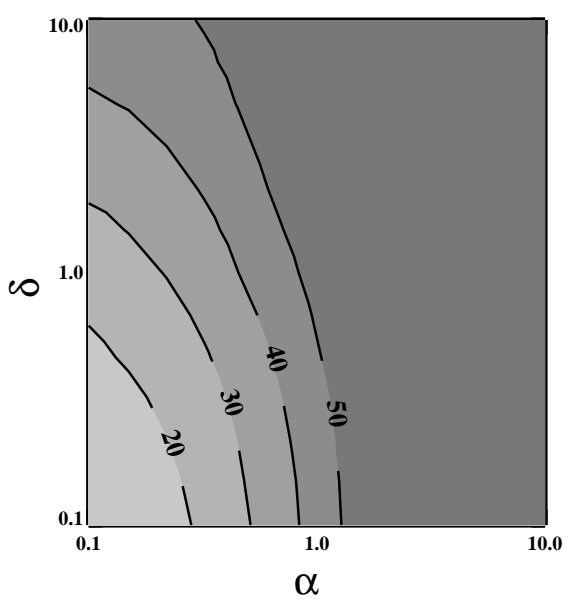

Fig. 3. Percentage discrepancies in results given by approximate method for $\tau=1.0$. a) Discrepancy in $x\left(\mathrm{X}_{2}\right)$; b) discrepancy in $x(\mathrm{XY})$; c) discrepancy in $x\left(\mathrm{Y}_{2}\right)$. Darker regions indicate greater discrepancies.

The inaccuracies in the results given by the modified rate equation approach are shown in Fig. 2 for $\tau=1.0$, and in Figs. $5 \mathrm{~d}-\mathrm{f}$ and $6 \mathrm{~d}-\mathrm{f}$. In most cases these results are no more accurate than those of the standard rate equation approach, and sometimes the accuracy is much less. Stantcheva et al. (2002) studied the system of $\mathrm{H}$ and $\mathrm{O}$ reacting on the surfaces of grains to form $\mathrm{H}_{2}, \mathrm{OH}$, and $\mathrm{O}_{2}$, and gave in their Figs. 1 to 3 
a)

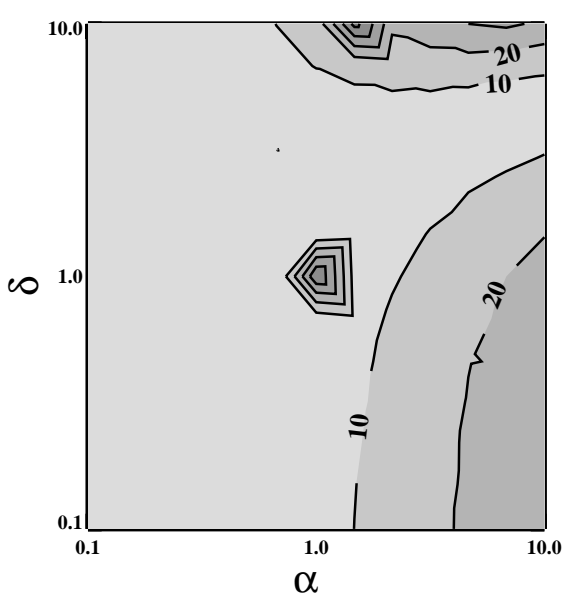

b)

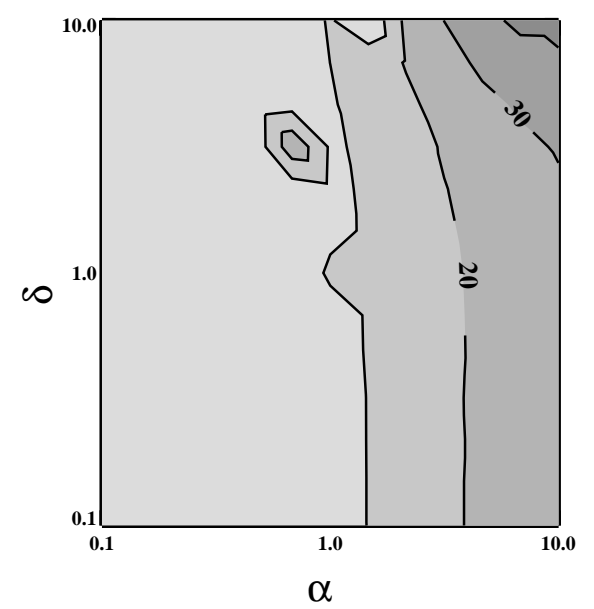

c)

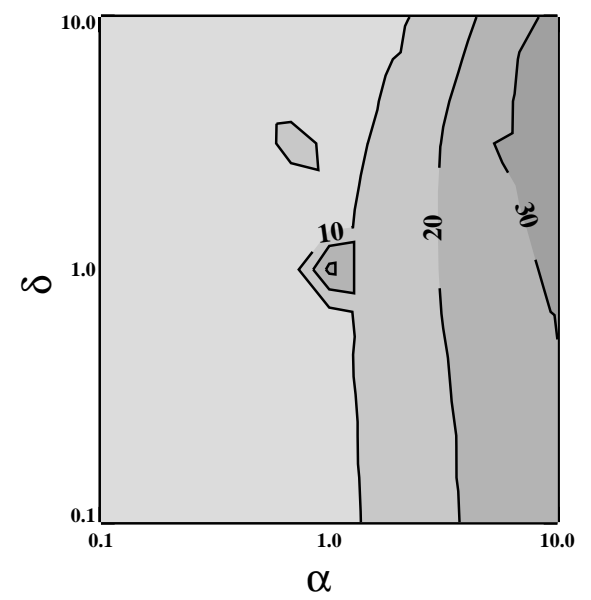

Fig. 4. Percentage discrepancies in results given by master equation approach with low truncation values for $\tau=1.0$. a) Discrepancy in $x\left(\mathrm{X}_{2}\right)$; b) discrepancy in $x(\mathrm{XY})$; c) discrepancy in $x\left(\mathrm{Y}_{2}\right)$. Darker regions indicate greater discrepancies.

results for the values of $x\left(\mathrm{H}_{2}\right), x(\mathrm{OH})$, and $x\left(\mathrm{O}_{2}\right)$ calculated by various different methods including the modified rate equation approach and the master equation method. The modified rate equation approach they used was the same as that used by Stantcheva et al. (2001), which was based on that used by Caselli et al. (1998). They found good agreement between results obtained with these two approaches for the parameters they considered, which were mostly rather different from those used by us. They used the same values for $a_{\mathrm{X}}, a_{\mathrm{Y}}, d_{\mathrm{X}}, t_{\mathrm{s}}(\mathrm{X})$, and $t_{\mathrm{s}}(\mathrm{Y})$ as Hasegawa et al. (1992), Caselli et al. (1998), and Green et al. (2001). The accretion rate they used for oxygen was different from that used for hydrogen. Their parameters were equivalent to $10^{-12}<a_{\mathrm{H}} t_{\mathrm{s}}(\mathrm{H})<10^{-5} ; a_{\mathrm{O}} t_{\mathrm{s}}(\mathrm{H}) \sim 10^{-10}$; $d_{\mathrm{H}} t_{\mathrm{s}}(\mathrm{H}) \sim 10^{-8}$; and $\frac{t_{\mathrm{s}}(\mathrm{O})}{t_{\mathrm{s}}(\mathrm{H})} \sim 10^{9}$. This corresponds to a region below and to the left of that shown in the plots in Fig. 2, and for a higher value of $\tau$. It can be seen that towards the bottom left-hand corner of Fig. 2a, the results are more accurate than elsewhere, which is consistent with the findings of Stantcheva et al. (2002).

Figure 3 shows that the approximate method gives inaccurate results for $x\left(\mathrm{X}_{2}\right)$ when $\delta$ is high. If the desorption rate is high, the assumption that an $\mathrm{X}$ atom will always react if it accretes onto a grain that is already populated is invalid. It was also found that there are inaccuracies in the values calculated for $x\left(\mathrm{Y}_{2}\right)$ for high $\alpha$, for all values of $\tau$. In these cases, the accretion rate is comparable to, or greater than, the rate of reaction of $\mathrm{Y}$, and the population of grains containing several $\mathrm{Y}$ atoms is non-negligible. At high values of $\tau$, the results were found to be inaccurate for a wide range of values of $\alpha$ and $\delta$, particularly for $x\left(\mathrm{X}_{2}\right)$ and $x\left(\mathrm{Y}_{2}\right) ; x(\mathrm{XY})$ is only inaccurate for high $\delta$ and low $\alpha$. The approximate equations were constructed under the assumption that if there are two atoms of species $\mathrm{Y}$ on the surface of a grain, and a third accretes, $\mathrm{Y}_{2}$ will not be formed. If we instead assume that a grain surface containing no atoms of $\mathrm{X}$ and more than two atoms of $\mathrm{Y}$ will always be a site of $\mathrm{Y}_{2}$ formation, Eq. (23) should be replaced with

$\Gamma\left(\mathrm{Y}_{2}\right) \approx \frac{\kappa_{\mathrm{Y}, \mathrm{Y}}+\alpha}{\kappa_{\mathrm{Y}, \mathrm{Y}}+2 \alpha} \frac{\alpha}{2} P(0,0)$.

In the case of $\alpha=0.3, \delta=0.1, \tau=10$, this results in an improvement of a factor of about 2.4 in the value obtained for the production rate of $\mathrm{Y}_{2}$. This results in $\mathrm{X}_{2}, \mathrm{XY}$, and $Y_{2}$ abundances, calculated with Eqs. (25) to (27), which differ from the exact results by $42 \%, 3 \%$, and $26 \%$, respectively; for $\mathrm{X}_{2}$ and $\mathrm{Y}_{2}$ this is a vast improvement from the results obtained when $\Gamma\left(\mathrm{Y}_{2}\right)$ was calculated with Eq. (23). The inaccuracy at high values of $\tau$ also causes an inaccuracy in $x\left(\mathrm{Y}_{2}\right)$ at high $\langle N(\mathrm{Y})\rangle$, which can be seen in Figs. 5i, and probably contributes to the inaccuracies seen in $x\left(\mathrm{X}_{2}\right)$ and $x(\mathrm{XY})$ in Figs. $5 \mathrm{~g}$ and $5 \mathrm{~h}$, at least at higher values of $\langle N(\mathrm{Y})\rangle$. In Fig. $5 \mathrm{~g}-\mathrm{i}$, one can see that the results given by this approach for $x\left(\mathrm{X}_{2}\right)$ are inaccurate for low $\langle N(\mathrm{X})\rangle$, at all values of $\langle N(\mathrm{Y})\rangle$; at higher values of $\langle N(\mathrm{Y})\rangle$ the results are inaccurate for a larger range of $\langle N(\mathrm{X})\rangle$. The results for $x(\mathrm{XY})$ are also inaccurate for low $\langle N(\mathrm{X})\rangle$ for a range of $\langle N(\mathrm{Y})\rangle$.

Figure 4 shows that the inaccuracy in the results given by the master equation method with low truncation values is high for high $\alpha$. In particular, the inaccuracy was found to be high in the case of high $\alpha$, low $\delta$, and low $\tau$. This corresponds to the case of a high rate of accretion, a low rate of $\mathrm{X}$ desorption, and high rates for reactions (2) and (3). This is the case in which there is a large population of $\mathrm{X}$ atoms on the surfaces of the grains. The inaccuracy is also high for high $\alpha$, high $\delta$, and high $\tau$, which corresponds to fast rates of accretion and 

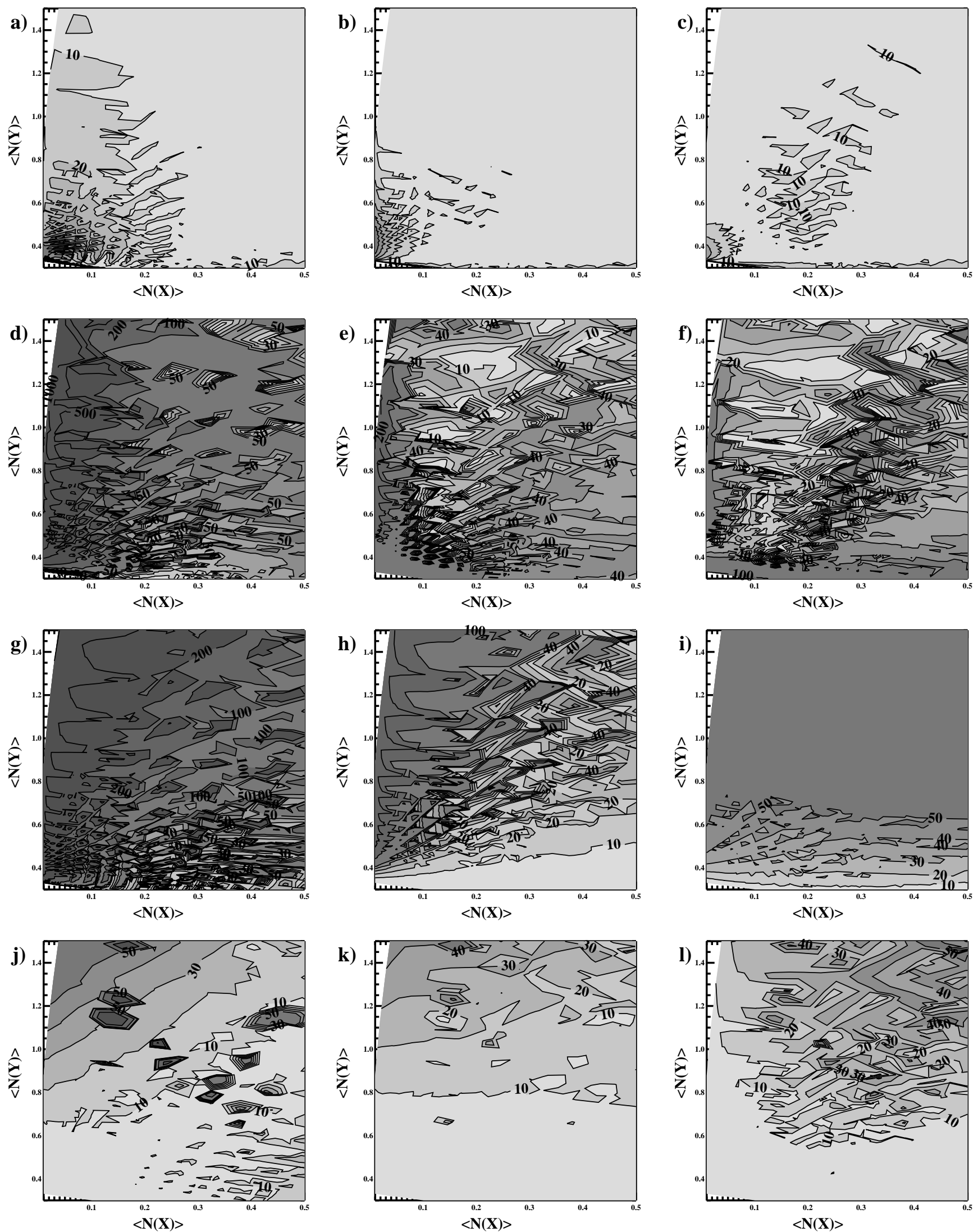

Fig. 5. Percentage discrepancies in results given by the various approaches, plotted against $\langle N(\mathrm{X})\rangle$ and $\langle N(\mathrm{Y})\rangle$ for all $\tau$. a)-c) standard rate equation approach; d)-f) modified rate equation approach; g)-i) approximate method; j)-l) low-truncation master equation approach. a), d), $\mathbf{g}$ ), and j) Discrepancy in $x\left(\mathrm{X}_{2}\right)$; b), e), h), and $\mathbf{k}$ ) discrepancy in $x(\mathrm{XY})$; c), f), i), and $\left.\mathbf{l}\right)$ discrepancy in $x\left(\mathrm{Y}_{2}\right)$. Darker regions indicate greater discrepancies. 

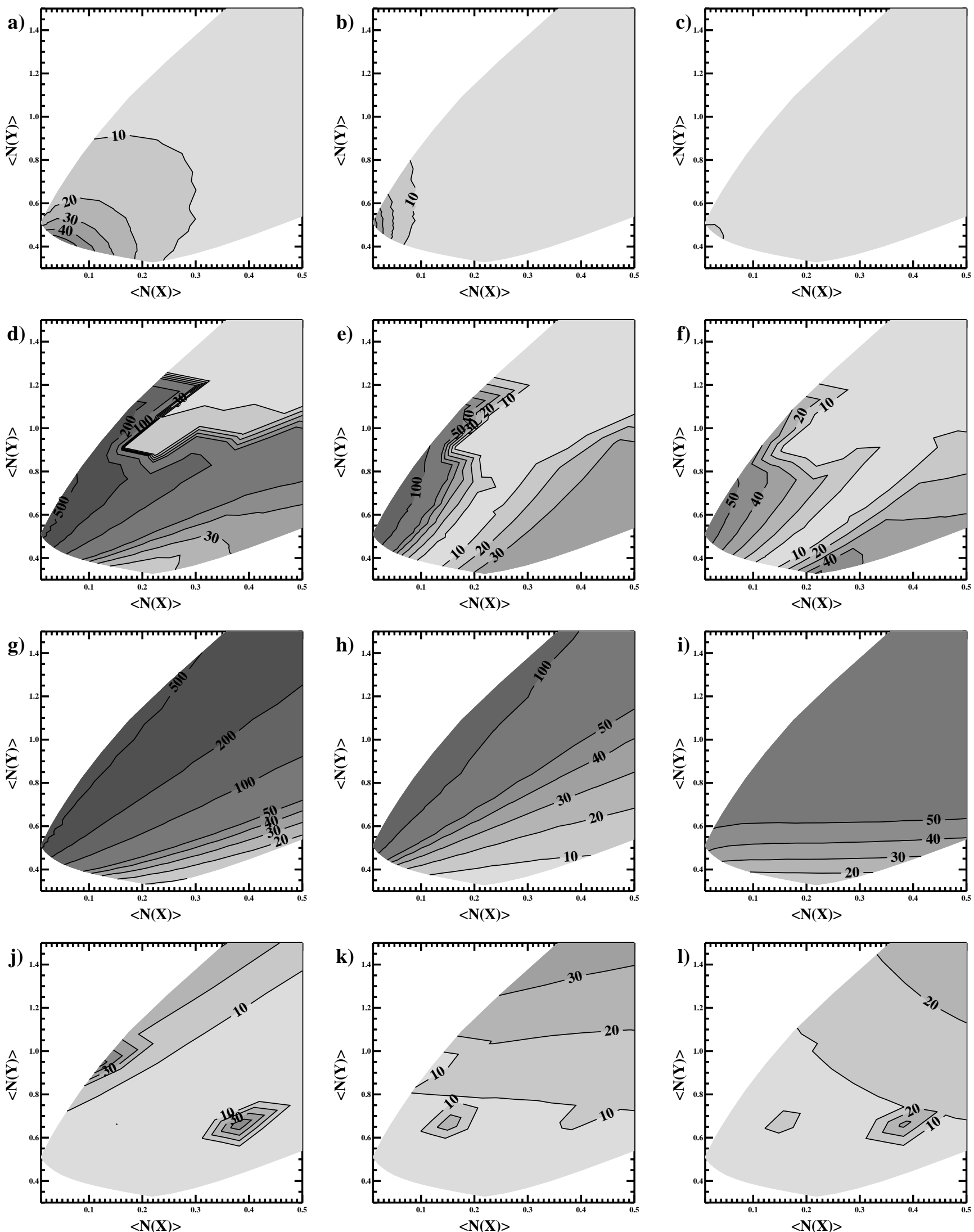

Fig. 6. Percentage discrepancies in results given by the various approaches, plotted against $\langle N(\mathrm{X})\rangle$ and $\langle N(\mathrm{Y})\rangle$ for $\tau=1$. a) $-\mathbf{c})$ Standard rate equation approach; d)-f) modified rate equation approach; g)-i) approximate method; j)-l) low-truncation master equation approach. a), d), $\mathbf{g})$, and j) Discrepancy in $x\left(\mathrm{X}_{2}\right)$; b), e), h), and $\left.\mathbf{k}\right)$ discrepancy in $x(\mathrm{XY})$; c), f), i), and $\left.\mathbf{l}\right)$ discrepancy in $x\left(\mathrm{Y}_{2}\right)$. Darker regions indicate greater discrepancies. 
$\mathrm{X}$ desorption, and a slow rate for reaction (3). This results in a large population of $\mathrm{Y}$ atoms on the surfaces of the grains. The results given by the master equation method with low truncation values are therefore inaccurate when there are large numbers of atoms on the surfaces of grains, as should be expected; this can also be seen in Figs. $5 \mathrm{j}-1$ and $6 \mathrm{j}-1$.

Figure 7 shows the regions of $\langle N(\mathrm{X})\rangle-\langle N(\mathrm{Y})\rangle$ space in which the standard rate equation approach, or the lowtruncation master equation approach, is more accurate. Plot a is for $\mathrm{X}_{2}$, plot $\mathrm{b}$ for $\mathrm{XY}$, and plot $\mathrm{c}$ for $\mathrm{Y}_{2}$. Dark regions indicate that the rate equation approach is more accurate; light regions are those in which the low-truncation master equation approach is more reliable. Regions of intermediate shading indicate that both approaches give results which are accurate to within $10 \%$. As expected, when either the results given by one method or both methods are not accurate to within $10 \%$, the low-truncation master equation approach is more accurate at low values of $\langle N(\mathrm{X})\rangle$ and $\langle N(\mathrm{Y})\rangle$, and the rate equation approch at higher values. The better of the two approaches gives results for $x\left(\mathrm{X}_{2}\right), x(\mathrm{XY})$, and $x\left(\mathrm{Y}_{2}\right)$ which are usually within 10 or $20 \%$ - and always within $25 \%$ - of those given by the exact method, except for the results given for $x\left(\mathrm{Y}_{2}\right)$ in a small region of $\alpha-\delta-\tau$ space around $\alpha=0.7,0.1<\delta<0.3, \tau>0.7$. In these cases, the more accurate method is the rate equation approach, and the value of $x\left(\mathrm{Y}_{2}\right)$ given by that method is around $40 \%$ less than the exact value.

Although in general the rate equation approach is more accurate than the low-truncation master equation approach for higher values of $\langle N(\mathrm{X})\rangle$ and $\langle N(\mathrm{Y})\rangle$, it can be seen in Fig. 7 that for $\langle N(\mathrm{X})\rangle \sim 1.2-1.4,\langle N(\mathrm{Y})\rangle \sim 3$, the low-truncation master equation approach is more accurate for $\mathrm{X}_{2}$. However, in most of these cases the results given by the two methods are very similar, and the discrepancies between them and the exact results are within about $20 \%$.

\section{Conclusions}

We have studied grain surface chemistry with a variety of approaches with a range of parameters. We deliberatelty made our study as general as was reasonable given the amount of work involved in a multiparameter study. We did so in anticipation of revisions of rates pertaining to specific problems treated in earlier literature, and of future studies of surface astrochemistry in a very wide range of environments including some in which atomic hydrogen accretion is unimportant.

The modified rate equation method was found to be no more accurate than the standard rate equation approach under the conditions examined; indeed it was in many cases less accurate. The modified rate equation method that we used is closely related to that of Caselli et al. (2002). They studied a system in which reaction barriers are important, and the values of $\alpha$ approriate for their studies are large. The modified rate equation approach that we used gives reliable results for large $\alpha$ for some values of $\tau$. Hence, our findings are in harmony with those of Caselli et al. (2002). The modified rate equation approach that we adopted differs from that employed by Stantcheva et al. (2001) which they found to give reliable results for the specific $\alpha \ll 0.1$ and large $\delta$ cases they examined. They, like the
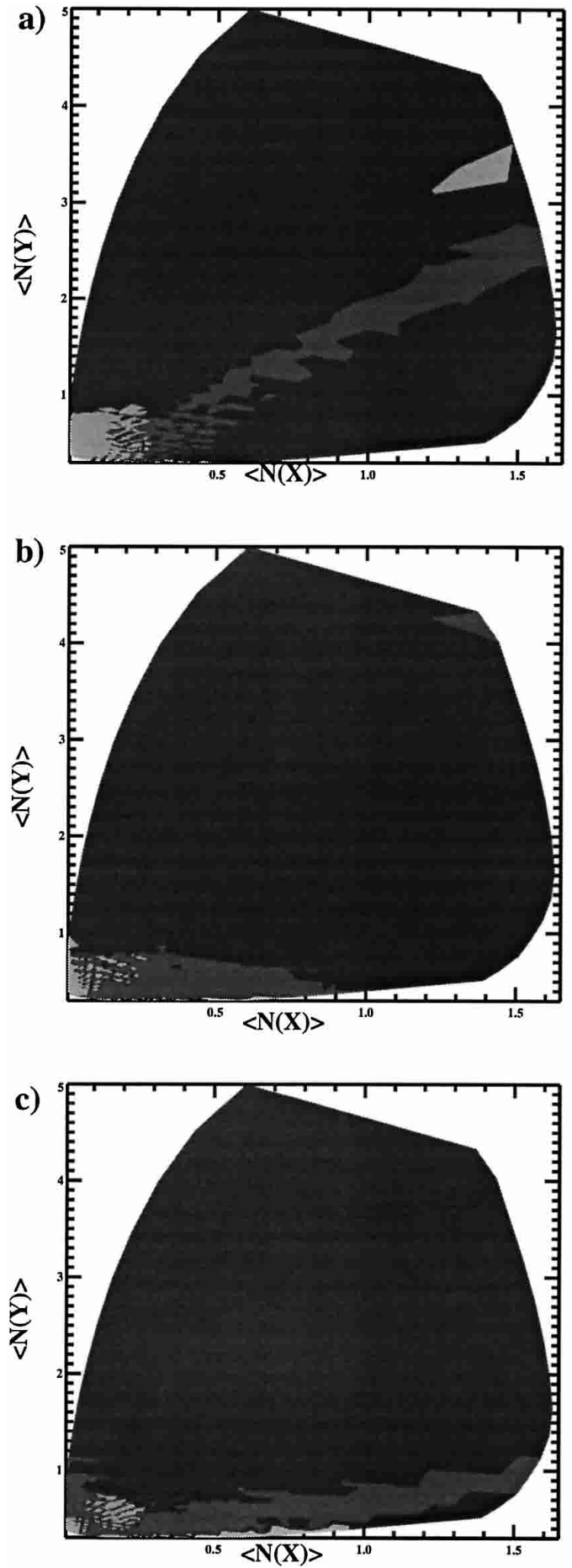

Fig. 7. Regions where results for a) $X_{2}$, b) $X Y$, and c) $Y_{2}$, given by standard rate equation approach (darker regions) and low-truncation master equation approach (lighter regions) are more accurate. Regions of intermediate shading are where both approaches give results which are accurate to within $10 \%$.

authors of a number of papers referenced here, were examining a region of parameter space they thought to be relevant when hydrogen accretion is important for the surface chemistry and the surface reactions proceed without barriers. Of the approximate methods we have examined, only the low-order truncated 
master equation approach is reliable for general small $\alpha$, large $\delta$ cases.

The approximate method of Green et al. (2001), which was devised specifically for the $\mathrm{H}, \mathrm{O}$ system, was also found to give inaccurate results under certain conditions, but can be improved by considering the possibility that an atom of species $\mathrm{Y}$ may accrete onto the surface of a grain on which there are already two $\mathrm{Y}$ atoms.

The standard rate equation approach was found to work well except under conditions which lead to there being a small average number of $\mathrm{X}$ and $\mathrm{Y}$ atoms on the surface of a grain. Under these conditions, the master equation approach with low truncation values is accurate. Therefore, a combination of the two approaches should be enough for a reasonably accurate calculation of the $\mathrm{X}_{2}, \mathrm{XY}$, and $\mathrm{Y}_{2}$ abundances in the parameter regime we considered - generally within about $20 \%$ of the exact values. Practical approaches to the use of stochastic models are under investigation (Stantcheva et al. 2001).

Acknowledgements. JGLR was supported by grants from the Leverhulme Trust and PPARC. TT was supported by a grant from PPARC.

\section{References}

Allen, M., \& Robinson, G. W. 1977, ApJ, 212, 396

Biham, O., Furman, I., Pirronello, V., \& Vidali, G. 2001, ApJ, 553, 595

Caselli, P., Hasegawa, T. I., \& Herbst, E. 1998, ApJ 495, 309

Caselli, P., Stantcheva, T., Shalabiea, O., et al. 2002, Plan. Space Sci., 50,1257

Charnley, S. B. 1998, ApJ, 509, L121

Charnley, S. B. 2001, ApJ, 562, L99

Charnley, S. B., Tielens, A. G. G. M., \& Rodgers, S. D. 1997, ApJ, 482, L203

Green, N. J. B., Toniazzo, T., Pilling, M. J., et al. 2001, A\&A, 375, 1111

Hasegawa, T. I., Herbst, E., \& Leung, C. M. 1992, ApJS, 82, 167

Pickles, J. B., \& Williams, D. A. 1977, Ap\&SS, 52, 433

Shalabiea, O. M., Caselli, P., \& Herbst, E. 1998, ApJ, 502, 652

Stantcheva, T., Caselli, P., \& Herbst, E. 2001, A\&A, 375, 673

Stantcheva, T., Shematovich, V. I., \& Herbst, E. 2002, A\&A, 391, 1069

Tielens, A. G. G. M., \& Allamandola, L. J. 1987, in ed. D. J. Hollenbach, \& H. A. Thronson, Interstellar Processes (Dordrecht: Reidel), 397

Tielens, A. G. G. M., \& Charnley, S. B. 1997, Origins Life Evol.

Tielens, A. G. G. M., \& Hagen, W. 1982, A\&A, 114, 245 